\title{
Implementation of Surface Radiation and Fluid-Structure Thermal Coupling in Atmospheric Reentry
}

\author{
Ojas Joshi and Pénélope Leyland \\ Ecole Polytechnique Fédérale de Lausanne, Interdisciplinary Aerodynamics Group, Station 9, 1015 Lausanne, Switzerland \\ Correspondence should be addressed to Ojas Joshi, ojas.joshi@epfl.ch
}

Received 20 October 2011; Revised 16 January 2012; Accepted 24 January 2012

Academic Editor: Roger L. Davis

Copyright ( 2012 O. Joshi and P. Leyland. This is an open access article distributed under the Creative Commons Attribution License, which permits unrestricted use, distribution, and reproduction in any medium, provided the original work is properly cited.

During atmospheric reentry, radiative heating is one of the most important component of the total heat flux. In this paper, we investigate how the thermal radiation coming from the postshock region interacts with the spacecraft structure. A model that takes into account the radiation reflected by the surface is developed and implemented in a solid solver. A partitioned algorithm performs the coupling between the fluid and the solid thermal fields. Numerical simulation of a hollow cone head and a deployed flap region shows the effects of the radiative cooling and the significance of the surface radiation.

\section{Introduction}

When a space vehicle travels through the different layers of the atmosphere in the reentry phase, its most external part is subject to extreme thermal conditions. A thermal protection system (TPS) is hence installed to insulate the vehicle's parts or its contents from high temperatures and heat fluxes. A significant amount of the total heat flux reaching the vehicle is due to the radiation coming from the postshock hot plasma [1].

Numerical simulations are one of the most widely used tools to design the TPS, and a detailed mathematical model allows to reduce margins in the sizing. A higher level of accuracy is obtained by including in the physical model the thermal response of the solid to the heat fluxes, through a coupled approach. In the latter, atmosphere gases and the spacecraft structure form two distinct systems that interact through the external surface of the TPS, and through which energy exchanges occur. To enhance the thermal control, the material of the TPS is chosen such that it reflects part of the incident radiation. However, in particular geometric configurations such as cavities, the reflected radiation can reach other surfaces of the vehicle structure. This paper exposes the development and the implementation of an implicit method for the calculation of surface radiation. This solution of the structure problem is coupled to the fluid flow through a partitioned algorithm.

Some space vehicles can be equipped with side flaps that are used to steer the vehicle. The deployed configuration of these flaps presents a gap, and radiative effects in this area are of major interest [2]. We will apply the methods developed in the first part to this particular geometry, and the effects of surface-to-surface radiation will be investigated.

\section{Numerical Tools}

During atmospheric reentry, the hot gas around the spacecraft transfers energy fluxes to the structure through the fluid-structure interface. The solid then reacts, with part of the heat being conducted in the deeper layers of the solid, and part being reradiated from the surface into the fluid. A continuous exchange is hence established on a transient basis, with a thermal energy equilibrium on the interface. The solution of the coupled problem is obtained using the domain decomposition approach, where each domain is described by its own model, and coupling conditions are defined on the boundary [3]. 
2.1. Fluid Domain Solver. The flow-field solver code eilmer3, developed by the Centre for Hypersonics (The University of Queensland, Australia), solves the compressible Navier-Stokes equations via a cell-centred time-dependent finite-volume formulation. The governing equations are expressed in integral form over arbitrary quadrilateral cells with the time rate of change of conserved quantities in each cell specified as a summation of the mass, momentum, and energy flux through the cell interfaces. A single temperature $T$ defines the population distributions amongst all thermal modes [4].

The conservation of mass, momentum, and total energy is expressed as

$$
\begin{gathered}
\frac{\partial \rho}{\partial t}+\sum_{j=1}^{d} \frac{\partial}{\partial x_{j}}\left(\rho u_{j}\right)=0 \\
\frac{\partial\left(\rho u_{i}\right)}{\partial t}+\sum_{j=1}^{d} \frac{\partial}{\partial x_{j}}\left[\left(\rho u_{i} u_{j}+\delta_{i j} p\right)-\tau_{i j}\right]=0 \\
\frac{\partial(\rho E)}{\partial t}+\sum_{j=1}^{d} \frac{\partial}{\partial x_{j}}\left[\left(\rho H u_{j}\right)-\sum_{i=1}^{d} u_{i} \tau_{i j}-\kappa \frac{\partial T}{\partial x_{j}}\right]=0 .
\end{gathered}
$$

In (1a)-(1c), $\rho$ denotes the total mass density, and $u$ is the average fluid velocity. The viscous stresses for a Newtonian fluid are given by:

$$
\tau_{i j}=\mu\left(\frac{\partial u_{j}}{\partial x_{i}}+\frac{\partial u_{i}}{\partial x_{j}}\right)+\lambda \delta_{i j} \nabla \cdot \mathbf{u},
$$

where the secondary viscosity coefficient $\lambda$ is expressed in terms of the primary viscosity coefficient $\mu$ via Stokes hypothesis, $\lambda=-(2 / 3) \mu$.

In (1c), $E$ is the specific total energy defined as

$$
E=\frac{1}{2}|\mathbf{u}|^{2}+e,
$$

where $e$ is the specific internal energy, and $(1 / 2)|\mathbf{u}|^{2}$ is the specific kinetic energy. The specific total enthalpy is denoted as

$$
H=E+\frac{p}{\rho} .
$$

To complete the system, the conservation equations have to be supplemented by the equation of state that provides pressure as a function of density and temperature, that is,

$$
p=p(\rho, T)
$$

Finally, $d=1,2,3$ is the number of dimensions of the problem, and $\delta_{i j}$ is the Kronecker delta.

2.2. Solid Domain Solver. The in-house solid solver is a code implemented in $\mathrm{C}++$ based on the finite element method and solves the heat transfer problem by means of conduction within the solid, according to the following transient equation:

$$
\rho C_{p} \frac{\partial T}{\partial t}-\nabla\left(\kappa_{s} \nabla T\right)=0,
$$

where $\rho$ is the solid mean density, $C_{p}$ and $\kappa_{s}$ are respectively the specific heat capacity at constant pressure and the heat conduction coefficient of the material; $T$ is the temperature.

On the walls that are in contact with the fluid, we impose a Neumann boundary condition, that remains constant between two successive coupling times.

In the classical discretised FEM matrix formulation of (6), the solution vector of temperatures $\mathbf{T}$ at time $n+1$, where $T_{i}, i=1, \ldots, n_{v}$ is the temperature value at node $i$, is calculated by solving the matrix system:

$$
\mathbf{A T}^{n+1}=\mathbf{B T}^{n}+\mathbf{c},
$$

where $\mathbf{A}$ and $\mathbf{B}$ are matrices of order $n_{v} \times n_{v}$, where $n_{v}$ is the number of nodes of the structural mesh. The vector c contains the contribution of the Neumann boundary conditions.

2.3. Implementation of Surface Radiation Heat Flux. All forms of matter emit and exchange thermal radiation with their surroundings. Radiation heat transfer can occur in the absence of matter and so also without temperature gradients.

In our work, we model the radiative heat flux taking into account the received radiation from adjacent surfaces (irradiation), and the radiation reflected and then emitted by the surface.

Given two different bodies or a single concave one, the radiative heat fluxes between the surfaces that "see" each other is modeled under two hypotheses:

(i) the individual surfaces absorb, reflect, and emit diffusively, independently of the temperature and the spectrum;

(ii) the gas between two surfaces is treated as optically thin, that is, it does not interact with the radiation; this implies that the radiative heat exchange occurs only between surfaces.

The components of the total radiative heat flux incident on a surface are [5]

(1) total irradiation towards the surface, $G$;

(2) emission from the surface, given by the StefanBoltzmann law:

$$
\widehat{E}=\varepsilon \sigma\left(T^{4}-T_{\text {ref }}^{4}\right),
$$

where $\mathcal{\varepsilon}$ is the surface emissivity, $\sigma=5.670 \times$ $10^{-8}\left[\mathrm{~W} \mathrm{~m}^{-2} \mathrm{~K}^{-4}\right]$ is the Stefan-Boltzmann constant, and $T_{\text {ref }}$ is a reference temperature. In the applications studied in this work, $T_{\text {ref }}^{4} / T^{4} \approx 0$ [6]; therefore, we will use hereafter the simplified form $\widehat{E}=\varepsilon \sigma T^{4}$;

(3) reflected part of irradiation from the surface, $\hat{\rho} G$, where $\hat{\rho}$ is the material reflectivity. 




(a)

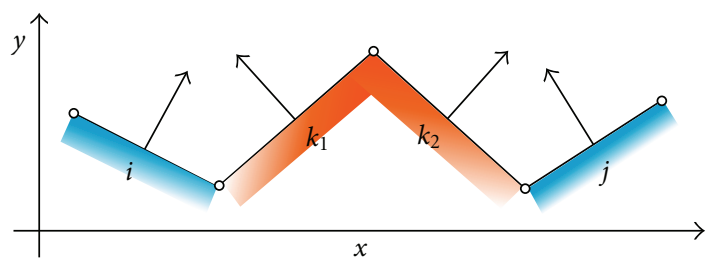

(b)

FIGURE 1: Two tests are performed to verify if two faces have a view factor $F_{i j} \neq 0$.

The sum of the last two components is called radiosity, denoted with $J$ [7], and represents the total radiation leaving a surface. Hence, we have

$$
J=E+\hat{\rho} G \text {. }
$$

In the computational domain, the solid boundaries are discretised into $n$ finite surfaces. For a given surface $i$, we can write the radiosity as

$$
J_{i}=\widehat{E}_{i}+\hat{\rho} G_{i}=\varepsilon \sigma T_{i}^{4}+\hat{\rho} \sum_{j=1}^{n} F_{i j} J_{j},
$$

where the so-called view factors $F_{i j}$ represent the ratio of diffuse energy leaving a surface $i$ received by a surface $j$ and the total diffuse energy leaving surface $i[8]$. This quantity is function of the angle formed by the normals of the two surfaces and varies between 0 (when two surfaces do not "see" each other) and 1 (when the surfaces are parallel and the line of sight is orthogonal). The computation of the view factor $F_{i j}$ is subsequent to the positive result of both the following tests:

(1) verify if the normal vectors to the two surfaces are intersecting (Figure 1(a));

(2) verify if no other surface $k$ is blocking the radiative heat transfer between $i$ and $j$ (Figure 1(b)). If any of the tests is negative, then $F_{i j}=0$.

We can express (10) in a matrix form for all the surfaces:

$$
\left(\begin{array}{cccc}
1 & -\hat{\rho} F_{12} & \ldots & -\hat{\rho} F_{1 n} \\
-\hat{\rho} F_{21} & 1 & \ldots & -\hat{\rho} F_{2 n} \\
\vdots & \vdots & \ddots & \vdots \\
-\hat{\rho} F_{n 1} & -\hat{\rho} F_{n 2} & \ldots & 1
\end{array}\right)\left(\begin{array}{c}
J_{1} \\
\vdots \\
\vdots \\
J_{n}
\end{array}\right)=\epsilon \sigma\left(\begin{array}{c}
T_{1}^{4} \\
\vdots \\
\vdots \\
T_{n}^{4}
\end{array}\right)
$$

or more compactly:

$$
\mathbf{R J}=\varepsilon \sigma \mathbf{T}^{4} .
$$

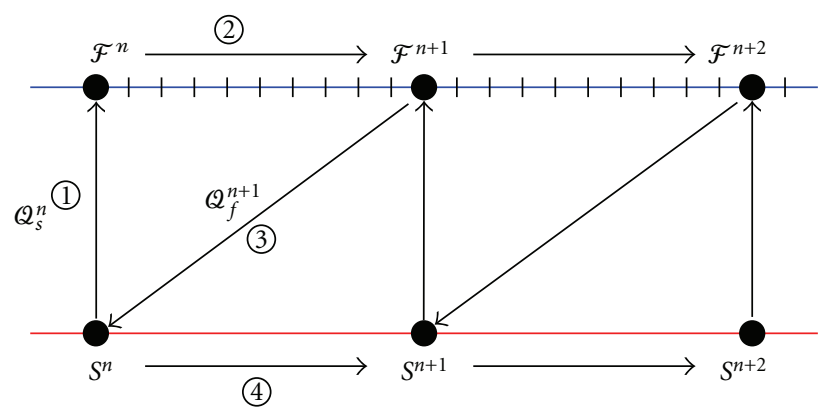

Figure 2: Partitioned coupling algorithm.

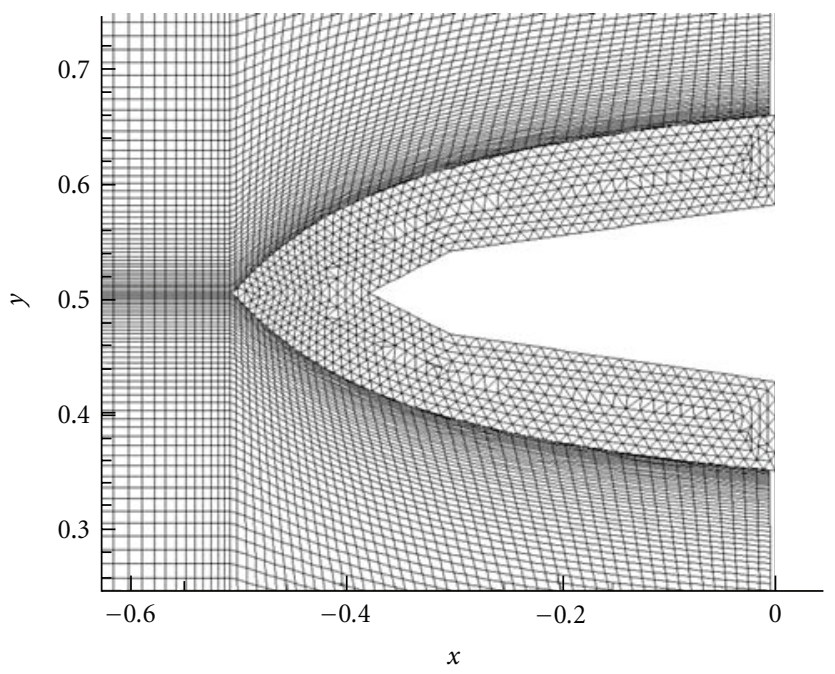

Figure 3: Fluid domain and structure shell meshes used for the simulations of the hollow cone head.

This system is solved for $\mathbf{J}$ using a Gauss elimination method and, once calculated the view factors matrix $\mathbf{F}$, the radiative heat flux contribution is given by:

$$
\mathbf{q}_{\mathrm{sr}}=\mathbf{J}-\mathbf{F J} \text {, }
$$

which is the source vector $\mathrm{c}$ in (7).

\section{Coupling Algorithm}

We consider a partitioned algorithm for fluid-structure interaction, that is, separate solvers are used for the fluid and the structure. This allows an independent time integration, and the coupling is obtained via a series of continuity relations at the interfaces and need to be solved iteratively.

The three elements of the solution of the coupled problem are then:

(1) solution of the fluid equations;

(2) solution of the heat transfer problem;

(3) definition of the solid-fluid interface conditions.

The thermal coupling between the fluid and the structure is given by the thermal equilibrium at the interface. The Dirichlet-Neumann method is used to impose continuity of 

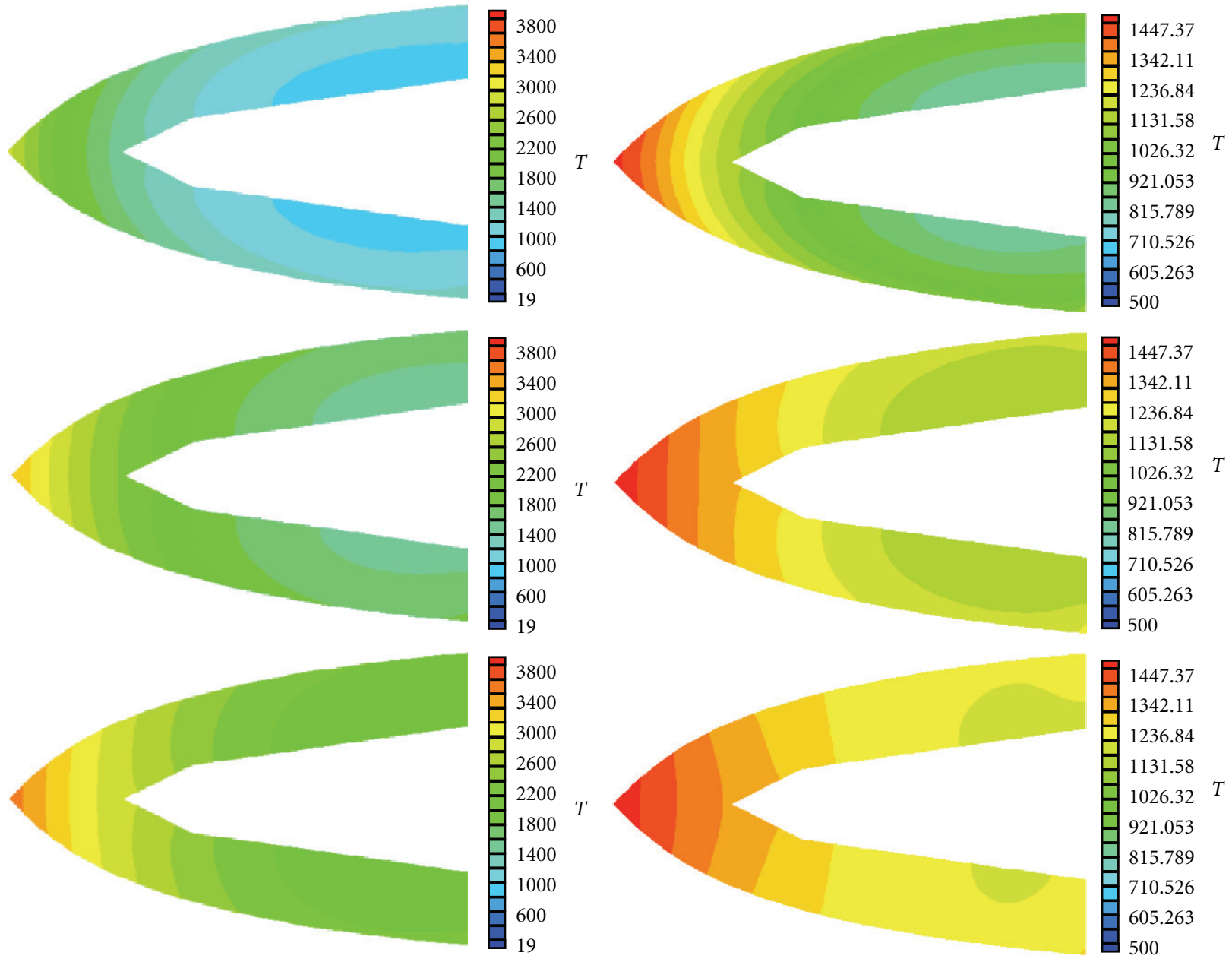

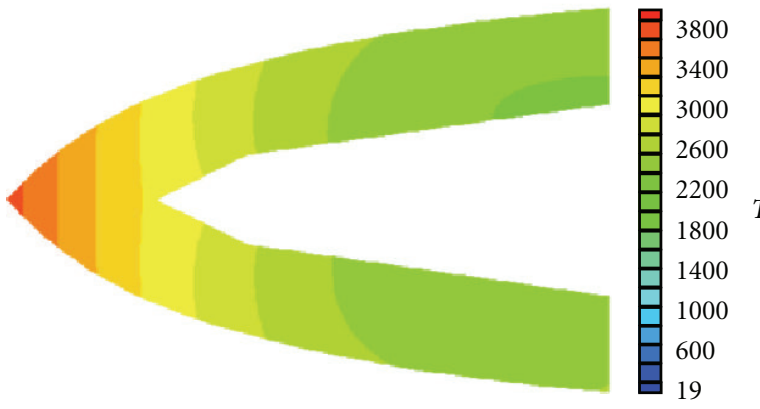

(a)

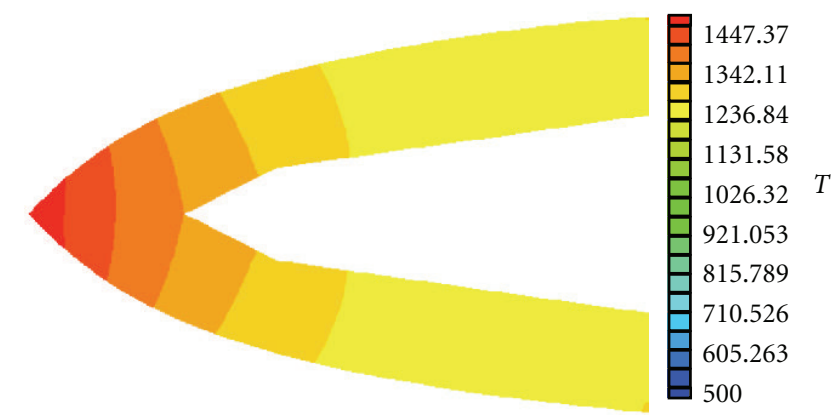

(b)

FIGURE 4: Temperature field within the hollow cone without (a) and with (b) radiation effects at time points $t=25 \mathrm{~s}, 50 \mathrm{~s}, 75 \mathrm{~s}$, and $100 \mathrm{~s}$.

temperatures and heat fluxes through the interface $\Sigma$. The coupling condition can then be written as:

$$
\begin{array}{ll}
T_{s}=T_{f} & \text { solid to fluid } \\
-\kappa_{f} \nabla T_{f} \cdot n=-\kappa_{s} \nabla T_{s} \cdot n+q_{\text {sr }} & \text { fluid to solid on } \Sigma,
\end{array}
$$

where $T_{s}$ and $T_{f}$ represent respectively the temperature in the solid and in the fluid, and $n$ is the local normal vector to each domain.

When we pass information from the fluid solver to the solid one, we impose a Neumann boundary condition on the interface $\Sigma$ of the solid domain. Contrariwise, we impose a Dirichlet condition on $\Sigma$ for the fluid domain when transferring information from the solid to the fluid.

We will denote with $Q_{f}=\left(T_{f}, q_{f}, \alpha_{s}\right)$ and $\mathcal{Q}_{s}=$ $\left(T_{s}, q_{s}, \alpha_{s}\right)$ the set of physical quantities at the interface, respectively from the fluid and the solid side. Supposing that at time $t^{n}$ the states of the fluid $\mathcal{F}^{n}$ and the structure $\delta^{n}$ are known, we want to determinate the states $\mathcal{F}^{n+1}$ and $f^{n+1}$ at time $t^{n+1}$.

The physical characteristic time scales of the aerofield and the heat transfer in the structure differ by many orders of 


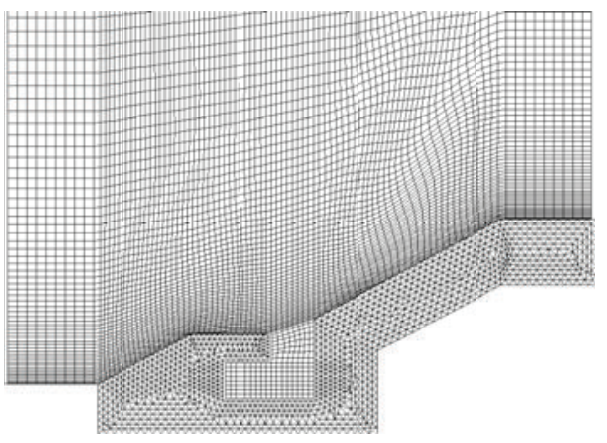

FIGURE 5: Mesh for the simulation of thermal coupling in a flap region.

magnitude, that is, $\tau_{\text {fluid }} / \tau_{\text {solid }} \approx 10^{9}$ [9]. The evolution of the temperature in the solid is extremely slow compared to that within the transient aerofield. This time scale difference reflects also in the numerical resolution of the problem, where the CFD code needs extremely small time steps, whereas we can allow a larger $\Delta t$ for the solid solver.

A loose coupling, however, does not guarantee conservation of the energy at the interface [10]. Moreover, also the convergence of this algorithm is not assured, especially in presence of high gradients $[11,12]$.

Taking this into account, we use in this work a strong coupling, that is, we subiterate interface data exchange until the residual of the coupled problem is less than a given threshold.

The algorithm develops as follows (see Figure 2)[13]:

(1) pass the solid interface physical quantities $\mathcal{Q}_{s}^{n}$ to the fluid solver;

(2) integrate the Navier-Stokes equations in time up to $t^{n+1}$ imposing as boundary condition the temperature on $\Sigma$ obtained from $\mathcal{Q}_{s}^{n}$;

(3) pass the fluid interface physical quantities $\mathcal{Q}_{f}^{n+1}$ to the heat equation solver;

(4) integrate the heat equation in time up to $t^{n+1}$ imposing as boundary condition the heat fluxes on $\Sigma$ obtained from $Q_{f}^{n+1}$;

(5) repeat steps (1) to (4) until convergence, then start next time step.

This kind of algorithm is computationally very expensive if the coupling is performed at every $\Delta t_{s} \approx \tau_{\text {solid. }}$. We therefore significantly reduced $\Delta t$ for the solid, and we have that step 2 in the previous algorithm is achieved with $N$ computational fluid time steps, while step 4 requires only one (see Figure 2).

Given the solvers we use, the computational domains are discretised according to the cell-centered finite volume and finite elements methods, respectively for the fluid and the solid domain. The junction is performed by matching the FE boundary nodes with the mid-points of the border sides of the FV cells.

\section{Results and Discussion}

TABLE 1: Cone head simulation parameters.

\begin{tabular}{lccc}
\hline \multicolumn{2}{c}{ Fluid } & \multicolumn{2}{c}{ Structure } \\
\hline$U_{\infty}$ & $3200 \mathrm{~ms}^{-1}$ & $\alpha_{s}$ & $2.5 \times 10^{-6} \mathrm{~m}^{2} \mathrm{~s}^{-1}$ \\
$M_{\infty}$ & 10 & $\kappa_{s}$ & $10 \mathrm{Wm}^{-1} \mathrm{~K}^{-1}$ \\
$P_{\infty}$ & $5000 \mathrm{~Pa}$ & $\hat{\rho}$ & 0.5 \\
$T_{\infty}$ & $250 \mathrm{~K}$ & $\epsilon$ & 0.5 \\
$\Delta t_{f}$ & $\propto 10^{-7} \mathrm{~s}$ & $T_{\text {initial }}$ & $300 \mathrm{~K}$ \\
$\delta_{m}$ & $\propto 1 \mu \mathrm{m}$ & $\Delta t_{s}$ & $\propto 10^{-3} \mathrm{~s}$ \\
Cells & 9600 & Elements & 1654 \\
\hline
\end{tabular}

4.1. Hollow Cone Head. A first evaluation of the coupling techniques is made on a simple hollow $0.5 \mathrm{~m}$ long cone head geometry immersed in air at high speed (Mach 10) in thermal and chemical equilibrium. The empty space inside the ogive is in vacuum conditions. We considered a very refractory material, with small values of thermal diffusivity $\alpha_{s}$ and conductivity $\kappa_{s}$. The values imposed for these parameters are not representative of a real material, but are functional for our analysis.

A significant issue in the simulations is to verify the importance of radiative heat transfer; hence, we simulate two cases: the first without and the second with surface reradiation. In the first case, the walls of the cone head are considered nonradiating, and we imposed an initial condition $T_{0}=300 \mathrm{~K}$ on these walls. On the other hand, when we take into account surface radiative heat transfer, the only heat flux on the external surfaces is of the type described by (13). In this case, surface radiative heat flux is formed only by emitted and reflected components, the view factor of all external surfaces being equal to 0 .

For all the simulations in this section, on the fluid domain we imposed supersonic inflow and outflow conditions, respectively on the left and right boundaries. Initial conditions are summarized in Table 1 . The geometry and the mesh is shown in Figure 3.

In Figures 4(a) and 4(b), the results of the computation are presented with and without radiation. When radiation effects are not taken into account, Figure 4(a), the surface temperature adapts progressively to the total temperature. Initially, the isothermal lines are parallel to the walls, indicating that heat transfer is almost equally important over the whole interface in contact with the fluid. As we advance in time, the isotherms become almost vertical, indicating that the heat propagates into the structure mostly from the nose tip, where temperature is at its maximum.

When surface reradiation is taken into account, the evolution of heat transfer in the structure changes considerably (Figure 4(b)). After an initial transient status, where the temperature field is similar to what seen before, the radiative cooling starts to become important. We notice that the isotherms are perpendicular to the structure external surface, and the temperature near the nose tip does not exceed $1550 \mathrm{~K}$, against almost $4000 \mathrm{~K}$ without radiation effects.

This simulation demonstrates that radiation between solid surfaces plays a considerable role, and the obtained 




(a)



(b)

Figure 6: Velocity of the flow inside the cavity at two random time points.
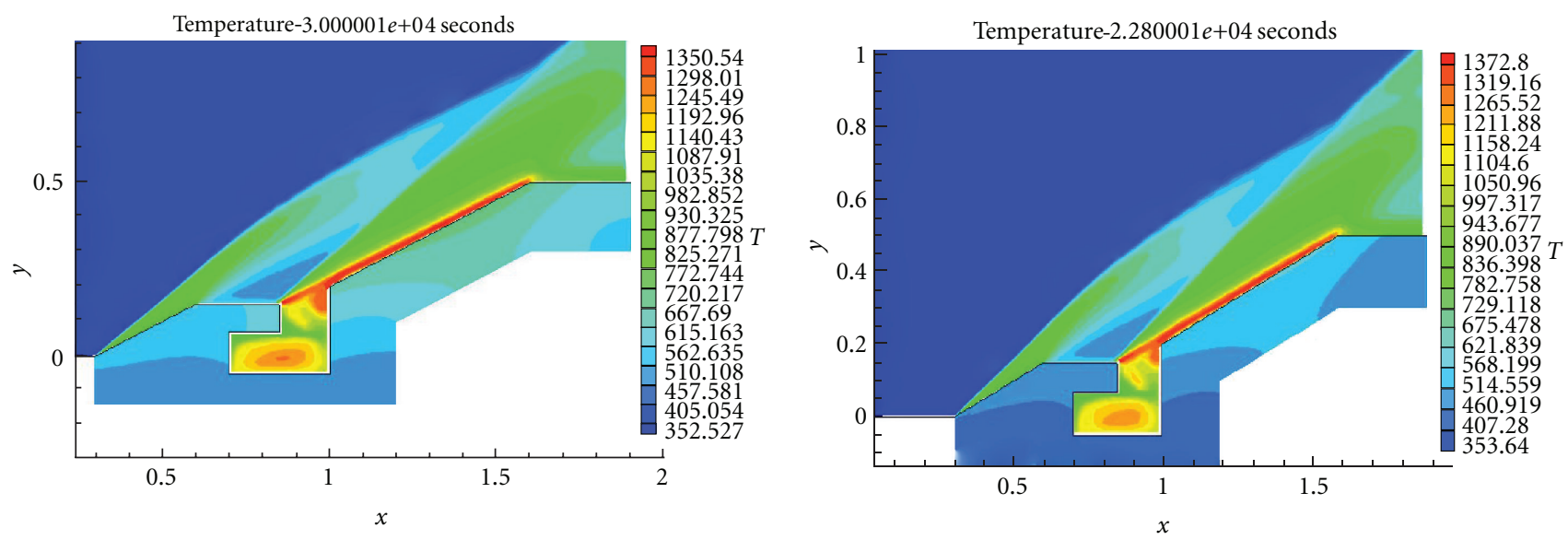

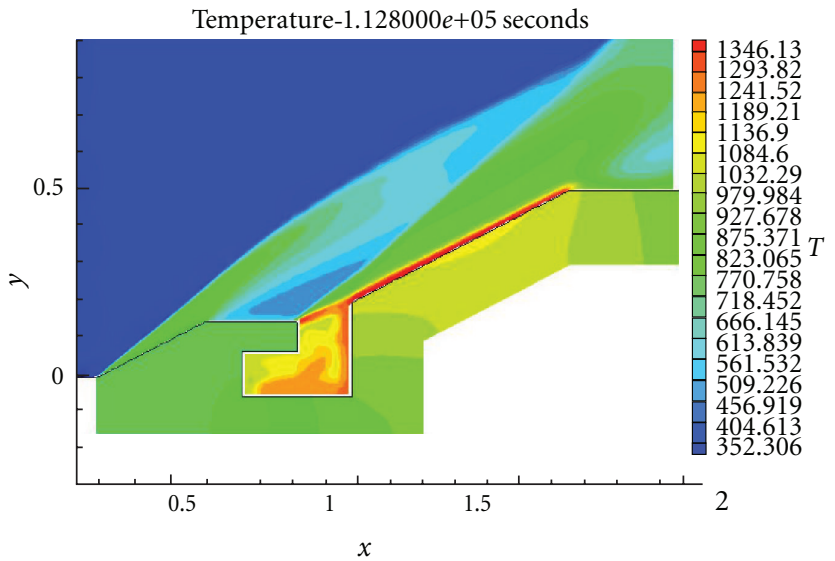

(a)

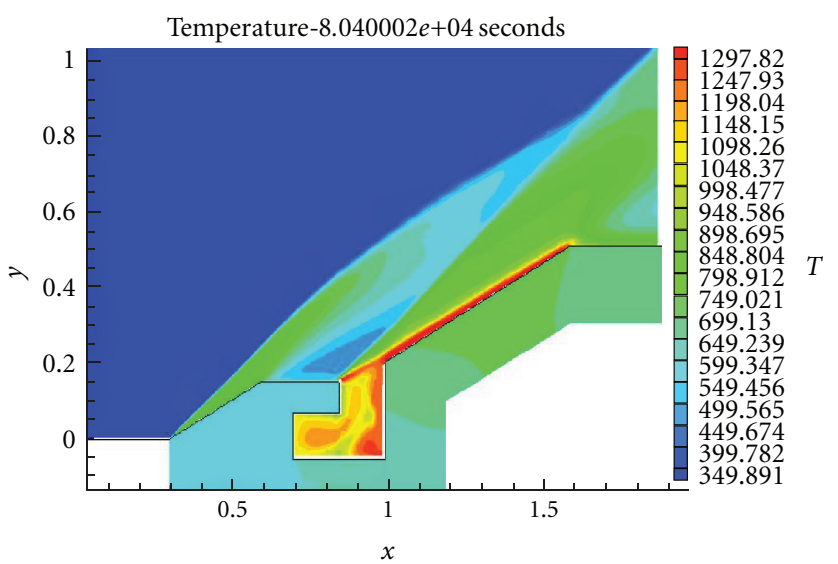

(b)

FIgURE 7: Temperature evolution inside and around a flap without (a) and with (b) radiation effects. 


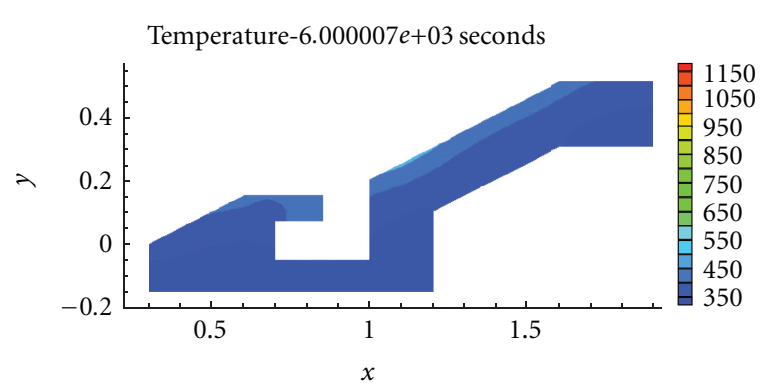

Temperature-6.600001e+04 seconds

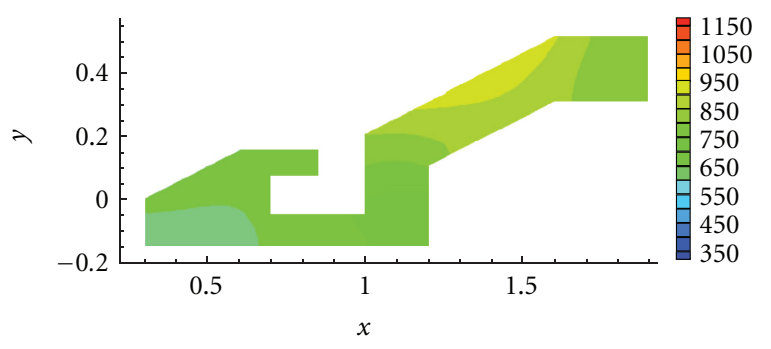

Temperature-1.284000e+05 seconds

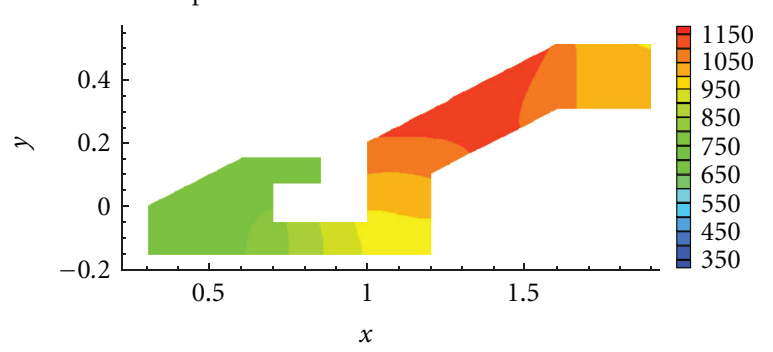

(a)

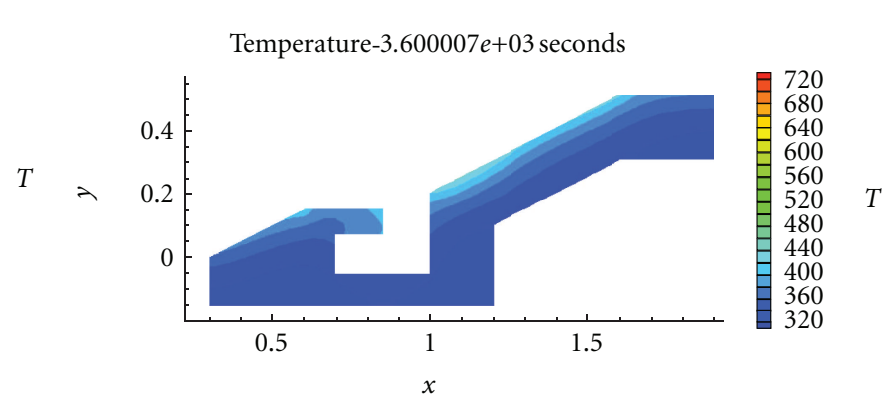

Temperature-5.040001e+04 seconds

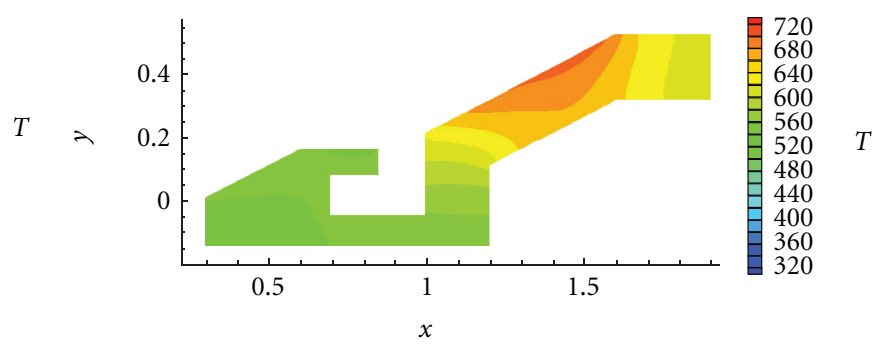

Temperature-9.000002e+04 seconds

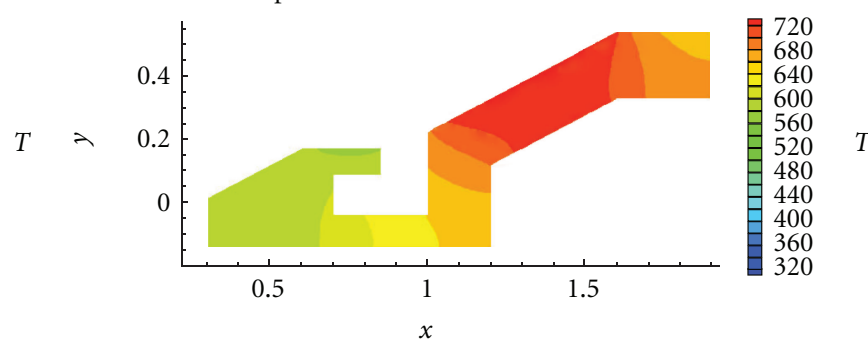

(b)

FIGURE 8: Evolution of the temperature within the flap without (a) and with (b) radiation effects.

temperature values, are of the same order of magnitude of those encountered in real applications, with such flow fields.

4.2. Deployed Flap Geometry. We consider now a more complex geometry: a model of a flap that, when deployed, creates a small cavity. The particularity of this case is that two surfaces of the same structure "see" each other, and the medium between these faces is a gas. We want to study the phenomena that take place in the cavity, as done in [2]. The method we use improves the evaluation of the radiation leaving the solid surface, by taking into account also reflected radiation. Moreover, as we have seen, we consider the surface radiation as a Neumann boundary condition for the solid domain.

We analyze here a cross-section of the flap. The total length of the flap is $0.4 \mathrm{~m}$, and base dimensions of the cavity are $0.075 \mathrm{~m}$ by $0.025 \mathrm{~m}$. The meshes used for these simulations are shown in Figure 5. The conditions are given in Table 2. The time steps are taken very small in order to properly capture instabilities and recirculation. Supersonic inflow and outflow conditions are imposed respectively on the left and right fluid boundaries. We consider the flap to be made of a very refractory material, with small values
TABLE 2: Flap simulation parameters.

\begin{tabular}{lccc}
\hline & Fluid & \multicolumn{2}{c}{ Structure } \\
\hline$U_{\infty}$ & $1700 \mathrm{~ms}^{-1}$ & $\alpha$ & $2.5 \times 10^{-6} \mathrm{~m}^{2} \mathrm{~s}^{-1}$ \\
$M_{\infty}$ & 5 & $\kappa_{s}$ & $10 \mathrm{Wm}^{-1} \mathrm{~K}^{-1}$ \\
$P_{\infty}$ & $50000 \mathrm{~Pa}$ & $\hat{\rho}$ & 0.5 \\
$T_{\infty}$ & $290 \mathrm{~K}$ & $\epsilon$ & 0.5 \\
$\Delta t_{f}$ & $\propto 10^{-8} \mathrm{~s}$ & $T_{\text {initial }}$ & $300 \mathrm{~K}$ \\
$\delta_{m}$ & $\propto 1 \mathrm{~mm}$ & $\Delta t_{s}$ & $\propto 10^{-4} \mathrm{~s}$ \\
Cells & 4705 & Elements & 2283 \\
\hline
\end{tabular}

of thermal diffusivity $\alpha_{s}$ and conductivity $\kappa_{s}$. The values imposed for these parameters are not representative [14] of a real material, but are functional for our analysis.

The external flow field is composed of a lip shock, a separation shock and a reattachment region as clearly shown within the solutions, Figures 7(a) and 7(b).

Before making any consideration from the thermal point of view, we notice from Figure 6 that at a random time point the flow speed and pressure in the cavity are very low. When the pressure rises, some air is ejected into the main flow field, and it acts as a continual pump. One effect of this low-speed 
region is that we have an important heating on the structure boundaries: we can observe from Figures 7(a) and 7(b) that the heat penetrates into the structure particularly from this region.

The temperature fields are shown at two instantaneous times without and with radiation respectively in Figures 8(a) and $8(\mathrm{~b})$. When taking into account radiation effects, we notice an overall lower temperature in the structure. The flap is still the prime site for heat absorption as well as the upper face of the cavity.

\section{Conclusions}

In this work, we defined a model of surface radiation and thermal interactions between atmospheric gases and the structure of a space vehicle in the phase of atmospheric entry. Because of this specific application, a special focus was made on radiation heat exchange. An efficient way of calculating the radiative heat flux and view factors has been devised and implemented. A mathematical formulation of the thermal coupling algorithm has been given. The consistent interface conditions are continuity of the temperature and heat flux within each one of the domains.

In the second part of this work, we focused on the realistic geometry of flap. The importance of radiative effects in this complex configuration has been analyzed. From the results we evince that, at such high temperatures, radiative heat transfer within the structure surfaces cannot be neglected. The coupled model shows the lowering of the temperature distributions due to radiation losses in the solid and gives a more reliable prediction of the thermal loads and their evolution. It is crucial to consider radiative heat transfer among surfaces in high-speed atmospheric entries, when even higher temperatures and heat loads than our case are attained.

The modelling here has been simplified and needs to be extended, especially for what concerns the physics and the chemistry of the flow and material properties.

\section{Acknowledgments}

This work was partially supported by the ESA-NPI Program, CCN C21872-1.

\section{References}

[1] J. D. Anderson Jr., Hypersonic and High Temperature Gas Dynamics, American Institute of Aeronautics and Astronautics, 2000.

[2] S. Borreca, F. Cipollini, G. Tumino, and J. M. Muylaert, "Fluidstructure thermal coupling on expert open flaps," in Proceedings of the 5th European Symposium on Aerothermodynamics for Space Vehicles, European Space Agency, Cologne, Germany, 2005.

[3] A. Quarteroni, Numerical Models for Differential Problems, Springer, 2009.

[4] P. A. Gnoffo, R. N. Gupta, and J. L. Shinn, "Conservation equations and physical models for hypersonic air flows in thermal and chemical nonequilibrium," NASA STI/Recon
Technical Report N 89:16115, 1989.

[5] F. P. Incropera and D. P. DeWitt, Fundamentals of Heat and Mass Transfer, Wiley, 5th edition, 2002.

[6] R. Siegel and J. R. Howell, Thermal Radiation Heat Transfer, Taylor \& Francis, 4th edition, 2002.

[7] C. M. Goral, K. E. Torrance, D. P. Greenberg, and B. Battaile, "Modeling the interaction of light between diffuse surfaces," Computer Graphics, vol. 18, no. 3, pp. 213-222, 1984.

[8] M. F. Modest, Radiative Heat Transfer, Academic Press, 2nd edition, 2003.

[9] E. Marín, "Characteristic dimensions for heat transfer," LatinAmerican Journal of Physics Education, vol. 4, no. 1, pp. 56-60, 2010.

[10] H. G. Matthies and J. Steindorf, "Partitioned strong coupling algorithms for fluid-structure interaction," Computers and Structures, vol. 81, no. 8-11, pp. 805-812, 2003.

[11] P. Le Tallec and J. Mouro, "Fluid structure interaction with large structural displacements," Computer Methods in Applied Mechanics and Engineering, vol. 190, no. 24-25, pp. 3039-3067, 2001.

[12] P. Leyland and V. Carstens, "Strongly coupled fluid structure interaction methods for flutter investigations in compressor cascades," in Fluid-Structure Interaction, Hermes, Paris, France, 2000.

[13] C. Farhat, K. G. van der Zee, and P. Geuzaine, "Provably second-order time-accurate loosely-coupled solution algorithms for transient nonlinear computational aeroelasticity," Computer Methods in Applied Mechanics and Engineering, vol. 195, no. 17-18, pp. 1973-2001, 2006, Fluid-Structure Interaction.

[14] M. Zingg, P. Leyland, and T. Ludwig, "Fluid-structure thermal coupling: application expert," in Proceedings of the International Conference East West HighSpeed Flow Field (EWHSFF '05), Beijing, China, 2005. 

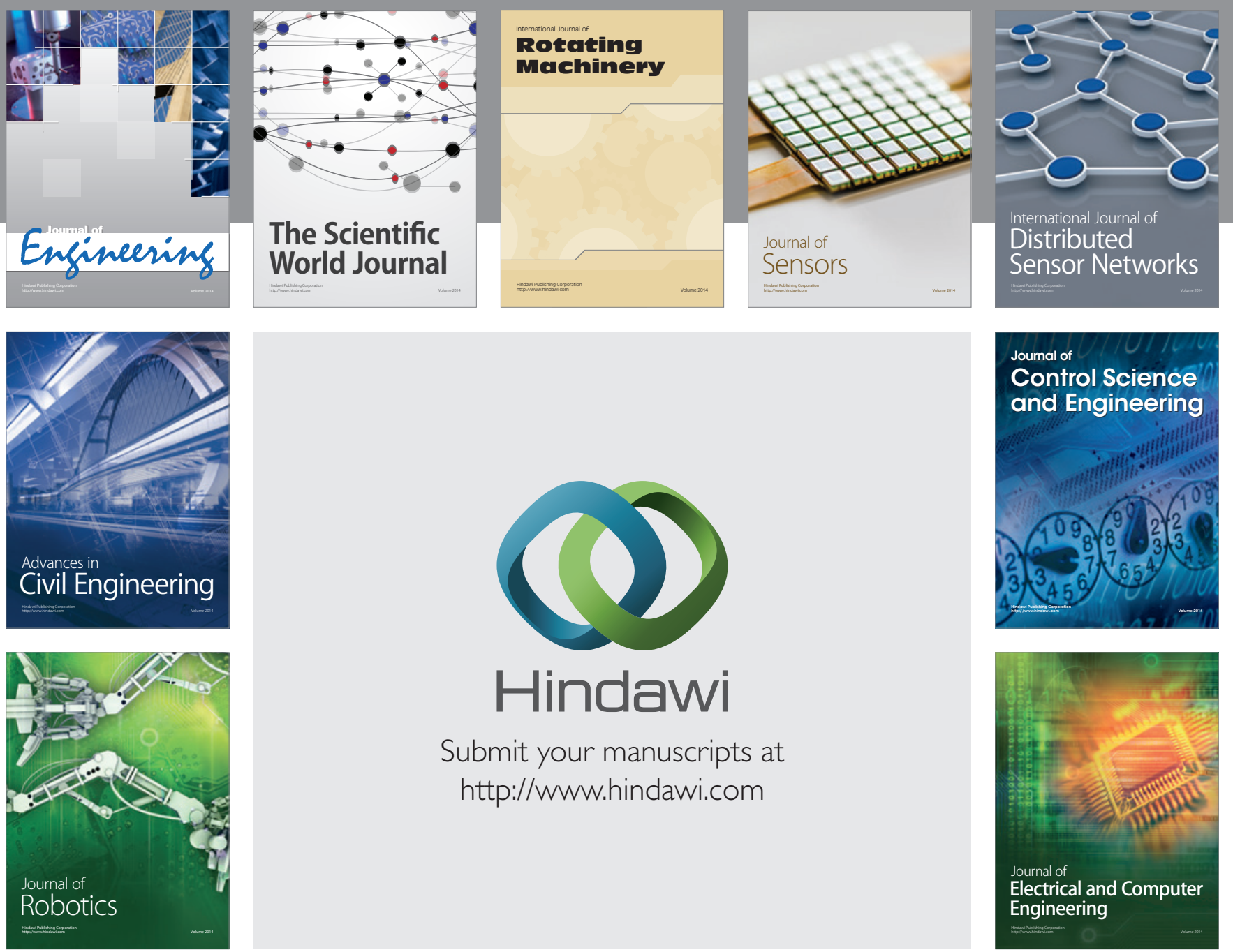

Submit your manuscripts at

http://www.hindawi.com
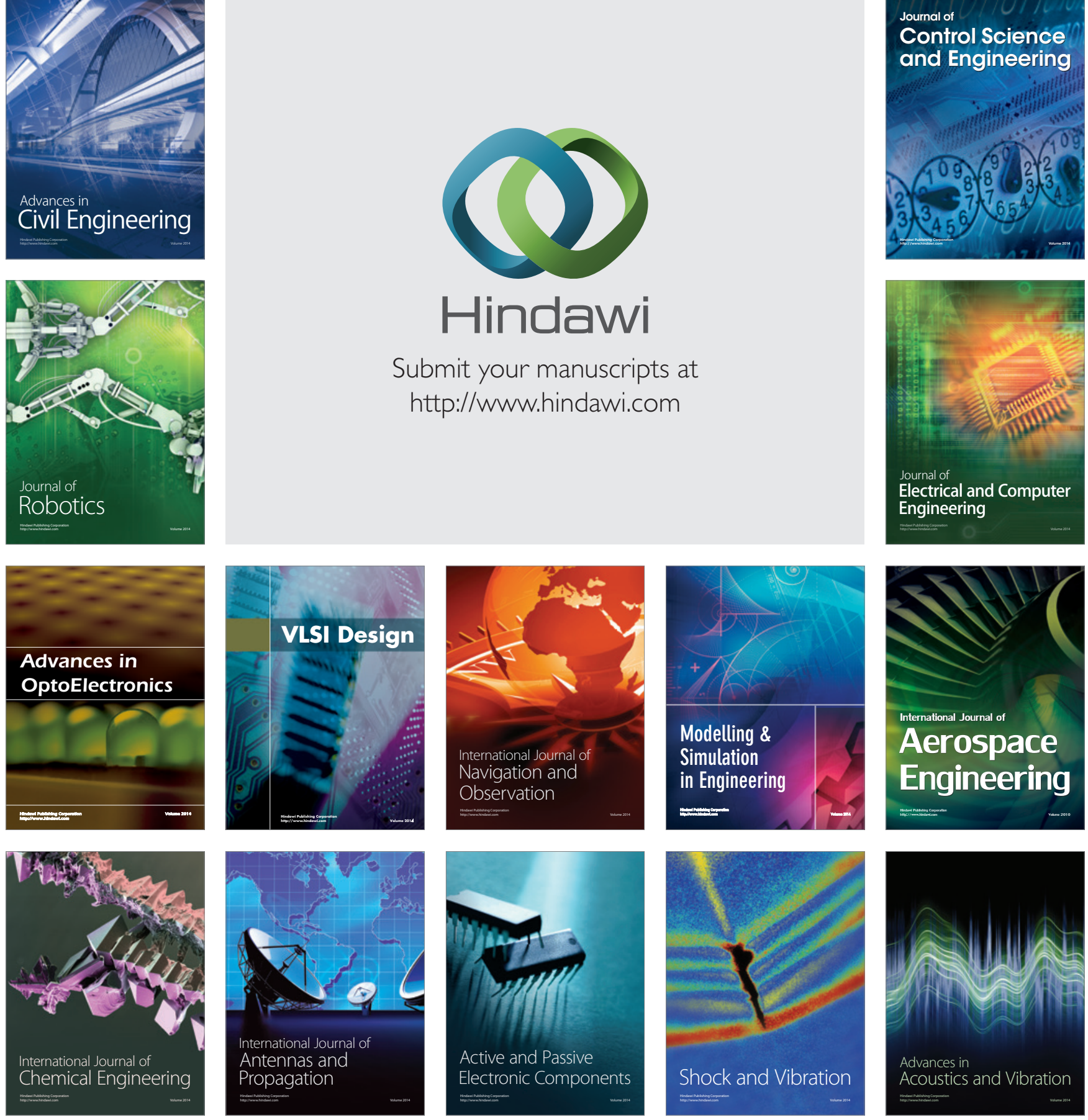